\title{
An INTEGRAL hard X-ray survey of the Large Magellanic Cloud ${ }^{\star}$
}

\author{
D. Götz ${ }^{1}$, S. Mereghetti ${ }^{1}$, D. Merlini ${ }^{1,2}$, L. Sidoli $^{1}$, and T. Belloni ${ }^{3}$
}

\author{
1 INAF - Istituto di Astrofisica Spaziale e Fisica Cosmica, via Bassini 15, 20133 Milano, Italy \\ e-mail: diego@iasf-milano.inaf.it \\ 2 Dipartimento di Fisica - Università degli Studi di Milano, via Celoria 16, 20133 Milano, Italy \\ 3 INAF - Osservatorio Astronomico di Brera, via E. Bianchi 46, 23807 Merate, Italy
}

Received 1 July 2005 / Accepted 27 October 2005

\section{ABSTRACT}

Two observation campaigns in 2003 and 2004 with the INTEGRAL satellite have provided the first sensitive survey of the Large Magellanic Cloud with an imaging instrument in the hard X-ray range ( $15 \mathrm{keV}-10 \mathrm{MeV})$. The high energy flux and long-term variability of the black hole candidate LMC X-1 was measured for the first time without contamination by the nearby $\left(\sim 25^{\prime}\right)$ young pulsar PSR B0540-69. We studied the accreting pulsar LMC X-4 by constraining the size of the hard X-ray emitting region $\left(\leq 3 \times 10^{10} \mathrm{~cm}\right)$ from analysis of its eclipses and by measuring its spin period $(13.497 \pm 0.005 \mathrm{~s})$ in the $20-40 \mathrm{keV}$ band. As it was in a soft state during the first observation and possibly in an extremely low state in the second one, LMC X-3 was not detected. Thanks to the large field of view of the IBIS instrument, we could also study other sources falling serendipitously in the observed sky region around the LMC: the Galactic low mass X-ray binary EXO 0748-676, the accreting pulsar SMC X-1 in the Small Magellanic Cloud, and the Active Galactic Nucleus IRAS 04575-7537. In addition we discovered five new hard X-ray sources, two of which most likely belong to the LMC.

Key words. gamma-rays: observations - pulsars: individual: PSR B0540-69 - X-rays: binaries - X-rays: galaxies - Magellanic Clouds

\section{Introduction}

The Large Magellanic Cloud (LMC) has been extensively observed in the soft X-ray range ( $E \leq 10 \mathrm{keV})$ with imaging X-ray satellites, which revealed a large number of X-ray sources of different classes: see e.g. Long et al. (1981) for Einstein, Sasaki et al. (2000), Haberl \& Pietsch (1999) for ROSAT, and Haberl et al. (2003) for XMM surveys. The brightest sources have also been observed at higher energies, but mostly with collimated hard X-ray detectors. Observations with non-imaging instruments include those performed with the $\mathrm{OSO}-7$ satellite, which yielded only upper limits for the LMC point sources (Markert \& Clark 1975), and with the Ginga satellite, which detected LMC X-3 (Ebisawa et al. 1993), LMC X-1 (Ebisawa et al. 1989), two black hole candidates, and LMC X-4, an accreting pulsar, up to $\sim 30 \mathrm{keV}$ (e.g. Levine et al. 1991). Both LMC X-3 and LMC X-1 were then studied with more sensitive instruments like HEXTE on board RXTE (e.g. Nowak et al. 2001) and the PDS on board BeppoSAX (Treves et al. 2000). However, all

\footnotetext{
* Based on observations with INTEGRAL, an ESA project with instruments and the science data centre funded by ESA member states (especially the PI countries: Denmark, France, Germany, Italy, Switzerland, Spain), Czech Republic and Poland, and with the participation of Russia and the USA.
}

these non-imaging instruments were affected by some confusion problems, due to source crowding in this region of the sky.

A few imaging surveys of this nearby $(d \sim 55 \mathrm{kpc})$ galaxy in the hard X-ray ( $E \geq 10 \mathrm{keV})$ energy range were carried out, though with limited sensitivity, exploiting coded mask telescopes. Observations with the SIGMA telescope (40-150 keV) on board the Granat satellite failed to reveal a significant hard X-ray flux from any of the high-energy sources of the LMC (Finoguenov et al. 1993). The data taken with ART-P, on board the same satellite, but working in a lower energy band (3-15 keV), revealed LMC X-1 and the young rotation powered pulsar PSR B0540-69, without any significant detection above $10 \mathrm{keV}$ (Grebenev et al. 1991). The TTM telescope (2-27 keV) detected only LMC X-4 and supernova SN 1987A above $15 \mathrm{keV}$ (Sunyaev et al. 1990, 1987).

Thanks to the good sensitivity and to the imaging capabilities of its instruments, the INTEGRAL observatory (Winkler et al. 2003) recently performed a deep observation of the LMC in the hard-X/soft- $\gamma$-ray domain. We will focus here on the results obtained with the ISGRI detector layer (15 keV-1 MeV, Lebrun et al. 2003) of the IBIS (Ubertini et al. 2003) coded mask telescope. Its large field of view $\left(\sim 29^{\circ} \times 29^{\circ}\right)$ contains the entire galaxy, and its good angular resolution $\left(\sim 12^{\prime}\right)$ is sufficient for resolving most of the LMC hard X-ray sources. 
Table 1. INTEGRAL LMC observations.

\begin{tabular}{ccc}
\hline \hline $\begin{array}{c}\text { INTEGRAL } \\
\text { Orbit }\end{array}$ & $\begin{array}{c}\text { Orbit Start } \\
\text { Time [UT] }\end{array}$ & $\begin{array}{c}\text { Net exposure } \\
{[\mathrm{ks}]}\end{array}$ \\
\hline 27 & 2003-01-02 04:07:37 & 209.2 \\
28 & 2003-01-05 03:53:52 & 71.0 \\
29 & 2003-01-08 03:41:54 & 212.0 \\
33 & $2003-01-2002: 53: 20$ & 216.2 \\
34 & $2003-01-2302: 38: 41$ & 215.1 \\
35 & $2003-01-2602: 25: 14$ & 217.2 \\
\hline $27-35$ & & 1140.7 \\
\hline 150 & $2004-01-0502: 11: 14$ & 80.3 \\
151 & $2004-01-0802: 00: 31$ & 197.3 \\
152 & $2004-01-1101: 49: 00$ & 130.3 \\
\hline $150-152$ & & 407.9 \\
\hline
\end{tabular}

\section{Observations and data analysis}

INTEGRAL performed two series of observations of the LMC. The first in January 2003, just after the end of the Performace and Verification phase of the satellite, and the second in January 2004. The dates and exposure times of the observations are reported in Table 1 . The highly inclined $\left(52^{\circ}\right)$ and long ( $72 \mathrm{~h}$ ) orbit of INTEGRAL allows for long, uninterrupted observations. However, in order to reduce the presence of the typical, systematic noise of coded mask instruments into the reconstructed sky images, each observation is divided in a series of individual pointings following a predefined pattern of sky directions around the target. The 2003 data were obtained in "hexagonal dithering" mode, which consists of the cyclic repetition of a pattern composed of 7 pointings, the first one aimed at SN 1987A and the following six spaced by two degrees at the vertices of a hexagon centred on the first pointing. The 2004 observation was instead performed using a dithering mode consisting of 25 pointings, spaced again by 2 degrees, arranged over a $5 \times 5$ square grid.

We analysed the IBIS data using version 4.2 of the Offline Scientific Software (OSA) provided by the INTEGRAL Science Data Centre (Courvoisier et al. 2003).

\subsection{Imaging}

After standard data processing (dead time correction, good time-interval selection, gain correction, energy reconstruction), we produced the sky images of each individual pointing in four energy bands: 20-40, 40-60, 60-100, and 100-150 keV. No sources are visible in the single pointings, owing to their short duration, with the exception of LMC X-4 (see below). To look for fainter sources, we produced the mosaics of the single orbits ( $200 \mathrm{ks})$ and of the two complete observations, after flat-fielding each individual sky image using the median of all the images of the same orbit. This procedure reduces the systematic noise induced by the non-uniformity of the detector shadowgrams, and thus improves the quality of the mosaic images.

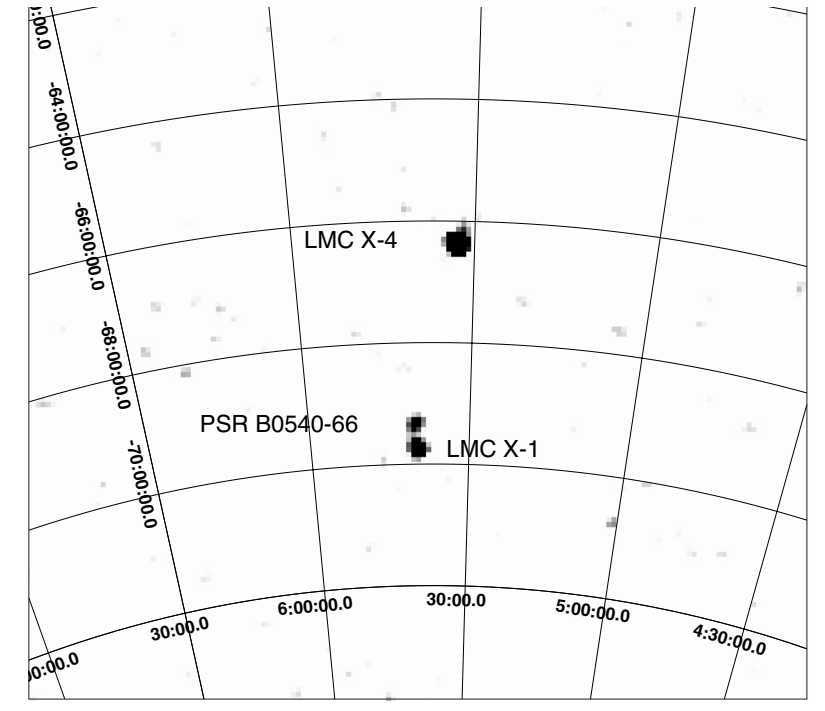

Fig. 1. IBIS/ISGRI image (20-40 keV) of the LMC region obtained in 2003. For the first time the flux of LMC X-1 can be clearly disentangled from the one of PSR B0540-69 at energies above $20 \mathrm{keV}$.

In the 20-40 keV mosaic of the 2003 observation we detected three LMC sources, LMC X-1, LMC X-4, and PSR B0540-69 (see Fig. 1), the Galactic low mass X-ray binary EXO 0748-676, and the Seyfert 2 galaxy IRAS 04575-7537. In the 2004 mosaic in the same energy band, we detected LMC X-4, PSR B0540-69, EXO 0748-676, and, at a large off-axis angle a source in the Small Magellanic Cloud, SMC X-1. Due to source variability, LMC X-1 was below the sensitivity threshold for the 2004 observation, which was too short to detect IRAS 04575-7537.

In addition to these known objects, we discovered a few new hard X-ray sources in the $20-40 \mathrm{keV}$ band. They were detected in the partial and total mosaics as indicated in Table 2. To evaluate the significance of their detection, we scaled the significance computed by the OSA software, to the $\sigma$ values derived by fitting a Gaussian to the pixel distribution of the final significance images. The coordinates, detection significance, and fluxes of the new sources are reported in Table 2. The uncertainty on their coordinates is about $3.5^{\prime}$.

\subsection{Spectral extraction}

To derive the average fluxes and spectra of each source we used the count rate values obtained from the mosaic sky images and rebinned the IBIS/ISGRI response matrix in order to match the four energy channels we used. This spectral extraction method is indicated for weak sources and has already been used successfully (see e.g. Mereghetti et al. 2005). In addition, we performed the imaging analysis and derived the spectra in nine energy bands between 20 and $200 \mathrm{keV}$ for the brightest sources (LMC X-4, LMC X-1 (only 2003 obs.), and EXO 0748-676). The spectra were fitted with XSPEC v 11.2 (Arnaud et al. 1996). A systematic error of $10 \%$ has been added to the data to account for the uncertainties of our spectral extraction method and of the response matrix. All the spectra were fitted with a power law model with the exception of LMC X-4 
Table 2. New sources detected during the LMC observation campaign.

\begin{tabular}{ccccccc}
\hline \hline Name (IGR) & $\begin{array}{c}\text { RA (J2000) } \\
\text { hh : mm : ss }\end{array}$ & $\begin{array}{c}\text { Dec (J2000) } \\
0^{\prime}{ }^{\prime}:{ }^{\prime \prime}\end{array}$ & $2003 \sigma$ & $\begin{array}{c}\text { 2003 Flux [mCrab] } \\
20-40 \mathrm{keV}\end{array}$ & $\begin{array}{c}\text { 2004 } \sigma \\
\text { 2004 Flux [mCrab] } \\
20-40 \mathrm{keV}\end{array}$ \\
\hline J03532-6829 & $03: 53: 14$ & $-68: 29: 00$ & 3.9 & 0.6 & - & - \\
J05009-7044 & $05: 00: 59$ & $-70: 44: 45$ & $4.1^{1}$ & 0.7 & - & - \\
J05319-6601 & $05: 31: 57$ & $-66: 01: 35$ & 6.2 & 0.9 & - & - \\
J05346-5759 & $05: 34: 35$ & $-57: 59: 35$ & 4.4 & 1.2 & - & 1.8 \\
J06239-6052 & $06: 23: 58$ & $-60: 52: 15$ & - & - & 4.6 & - \\
\hline
\end{tabular}

${ }^{1}$ Reaches $5.5 \sigma(2.5 \mathrm{mCrab})$ during orbit 27.

Table 3. Fluxes (20-100 keV) and spectral parameters derived for the sources detected by IBIS/ISGRI during the 2003 (upper panel) and 2004 (lower panel) observations. The errors are all at $1 \sigma$ level for one parameter of interest.

\begin{tabular}{c|c|c|c|c|c}
\hline \hline & LMC X-1 & LMC X-4 & PSR B0540-69 & EXO 0748-676 & IRAS 04575-7537 \\
Flux $\left[10^{-12} \mathrm{erg} \mathrm{cm}^{-2} \mathrm{~s}^{-1}\right]$ & $58_{-6}^{+8}$ & $124 \pm 60$ & $31_{-10}^{+14}$ & $320 \pm 150$ & $15.3 \pm 5.1$ \\
Photon Index $\Gamma$ & $2.9 \pm 0.3$ & $1.0 \pm 0.2$ & $2.2 \pm 0.4$ & $1.3 \pm 0.4$ & $3.4 \pm 1.0$ \\
Cutoff Energy $E_{c}[\mathrm{keV}]$ & - & $13 \pm 5$ & - & $44 \pm 15$ & - \\
\hline Flux $\left[10^{-12} \mathrm{erg} \mathrm{cm}^{-2} \mathrm{~s}^{-1}\right]$ & - & $590 \pm 100$ & $24 \pm 7$ & $421 \pm 150$ & - \\
Photon Index $\Gamma$ & - & $1.35 \pm 0.2$ & $1.9 \pm 0.5$ & $1.6 \pm 0.4$ & - \\
Cutoff Energy $E_{c}[\mathrm{keV}]$ & - & $17 \pm 7$ & - & $50 \pm 15$ & - \\
\hline
\end{tabular}

and EXO 0748-676, for which a cutoff power law $\left(E^{-\Gamma} \times\right.$ $\mathrm{e}^{\left(-E / E_{\mathrm{c}}\right)}$, where $E_{\mathrm{c}}$ is the cutoff energy and $\Gamma$ the photon index) gives better results. The photon spectra were then multiplied by a constant factor $(\sim 1.7)$ derived from comparison with observations of the Crab Nebula.

In order to look for spectral variability, we also considered the mosaics of the single orbits. With the exception of LMC X-4 (see below), all sources were consistent with a constant flux within the same observation, while, as mentioned above, some long term variations appeared by comparing the fluxes in 2003 and 2004 (LMC X-1). All the fluxes and spectral parameters, referring to the $20-100 \mathrm{keV}$ energy range, are reported in Table 3.

\subsection{Timing analysis}

\subsubsection{LMC X-4}

From the mosaics of the individual orbits we noticed that the flux of LMC X-4 increased by a factor $\sim 7$ in 6 days during the first part of the January 2003 observation, and returned to the initial level towards the end of the observation. This can be seen clearly from the light curve obtained from the fluxes measured in the individual pointings $(\sim 2200 \mathrm{~s}$ each, Fig. 2, upper panel). The source variability can be attributed to the 30.3-day super-orbital period observed at lower energies (e.g. Paul \& Kitamoto 2002) and generally interpreted as the effect of precession of a tilted accretion disk. The bottom panel of Fig. 2 shows for comparison the $2-10 \mathrm{keV}$ light curve obtained by folding the data of the RXTE ASM instrument at $P_{S}=$ 30.296 days. Our 2003 observation, extending over 26 days, covered most of the super-orbital period but missed the peak of the source intensity (top panel of Fig. 2), while a much shorter phase coverage was obtained in 2004 (Fig. 2 middle panel).

Referring to the upper panel of Fig. 2, we define as the high and low states of the source when the $20-40 \mathrm{keV}$ flux is above/below 4 counts/s. The spectra extracted over the two states differ only in flux (from $7 \times 10^{-11}$ to $4.3 \times$ $10^{-10} \mathrm{erg} \mathrm{cm}^{-2} \mathrm{~s}^{-1}$ ) but show a similar shape. For comparison we also extracted the high state spectra of LMC X-4 also using the standard OSA 4.2 spectral extraction, which is suitable for bright sources that are clearly detected during single pointings. In fact LMC X-4 was also detected in the single pointings in this case. The two methods gave similar results.

When the source was in the high state, the eclipses due to its occultation by the giant companion star $\left(P_{\text {orb }}=1.4084\right.$ days, White 1978) are visible in the INTEGRAL light curves. To study the eclipses in more detail, we extracted the light curves (20-40 keV) with $100 \mathrm{~s}$ resolution around the time of the eclipses, both for the 2003 and 2004 observations. We added the data of the two complete eclipses of the 2004 observation in phase; see Fig. 3. The eclipse ingress seems to be resolved, and in fact it cannot be fitted with a step function. The same is not true for the egress. Assuming that the ingress is truly resolved, we can derive some information on the size of the largest hard X-ray emitting region. If the geometry of the binary system and its projection on the sky are known, we can assume that at the beginning of the eclipse the projection of the distance between the centres of the two binary components coincides with the sum of their radii, while it coincides with their difference at the beginning of the total occultation. We indicate with $R_{\mathrm{S}}$ and $R_{\mathrm{X}}$ the radii of the companion star and the hard $\mathrm{X}$-ray emission region, respectively, with $R$ the binary separation $\left(8.5 \times 10^{11} \mathrm{~cm}\right.$, Levine et al. 2000) and with $\theta=2 \pi \phi$ the 


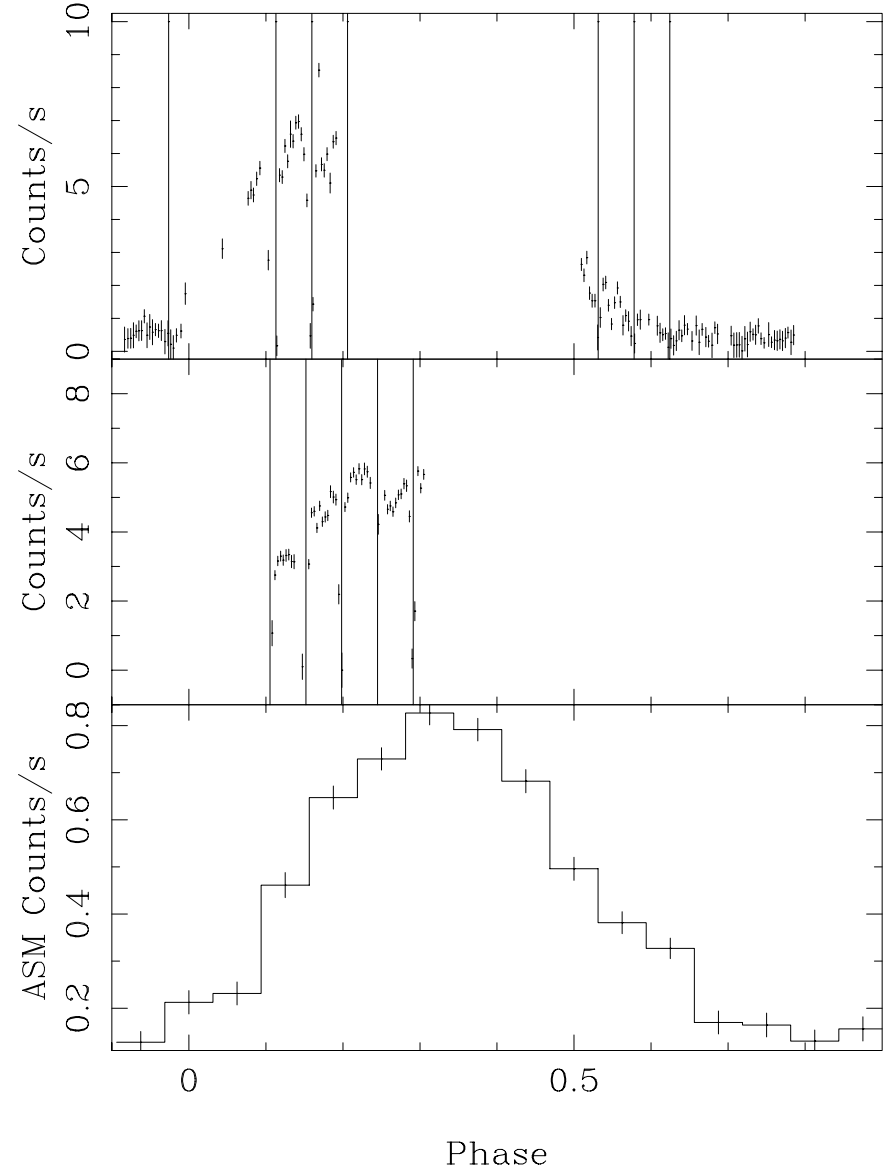

Fig. 2. Upper panel: IBIS/ISGRI light curve of LMC X-4 during the 2003 observation (20-40 keV). The time has been converted to superorbital phase (period $=30.3$ days), and the original data have been rebinned by a factor 4 ( $\sim 8800 \mathrm{~s}$ time bins). Middle panel: IBIS/ISGRI light curve of LMC X-4 during the 2004 observation (20-40 keV). The vertical lines represent the times of mid eclipse as derived by the ephemeris of Naik \& Paul (2004). Lower Panel: RXTE/ASM light curve of LMC X-4 folded at the super-orbital period. For all plots phase $=0$ corresponds to 52644 MJD.

phase ( $\theta_{1}$ for the early beginning and $\theta_{2}$ for the start of the total occultation) and obtain the following relations:

$R_{\mathrm{X}}+R_{\mathrm{S}}=R\left(\sin ^{2} \theta_{1}+\cos ^{2} \theta_{1} \cos ^{2} i\right)^{1 / 2}$

$R_{\mathrm{X}}=R_{\mathrm{S}}-R\left(\sin ^{2} \theta_{2}+\cos ^{2} \theta_{2} \cos ^{2} i\right)^{1 / 2}$

where $\theta=0$ corresponds to the conjunction of the two stars with the compact object closer to the observer, and $\theta=\pi$ corresponds to the mideclipse time. By fitting the light curve with a constant plus an exponential cutoff, one can derive precise values for $\theta_{1}=2.614$ and $\theta_{2}=2.714$. Substituting Eq. (1) in Eq. (2) and knowing the geometry of the system (inclination angle $i=68^{\circ}$, Levine et al. 1991), we derive a size for the high energy emitting region, $R_{\mathrm{X}}, 2.63 \times 10^{10} \mathrm{~cm}$.

We looked for the presence of the $\sim 13.5 \mathrm{~s}$ neutron star spin period by analysing those pointings during which the source was brighter. The photon arrival times were converted to the solar system barycentre and corrected for the orbital motion of the system. We restricted our search to the $15-40 \mathrm{keV}$ energy

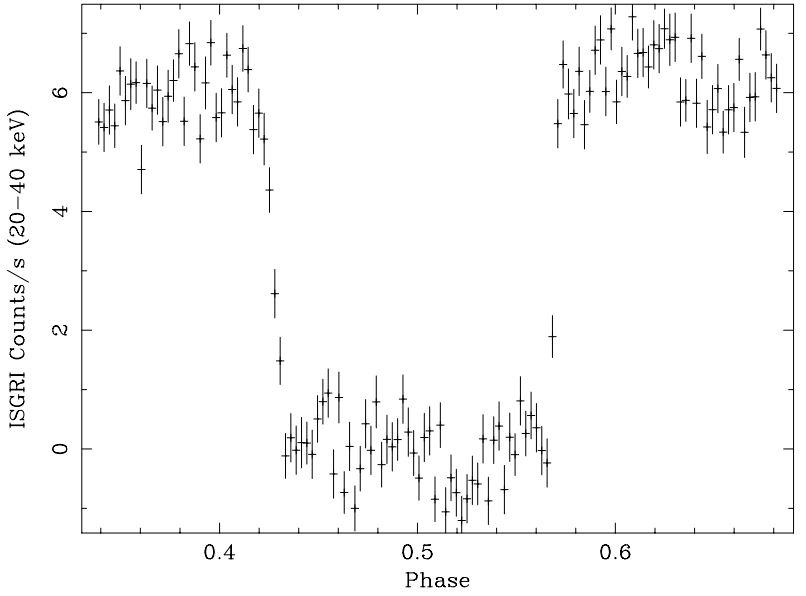

Fig. 3. IBIS/ISGRI (20-40 keV) average light curve of the two eclipses of LMC X-4 used in our analysis (see text). Time bin = $300 \mathrm{~s}$.

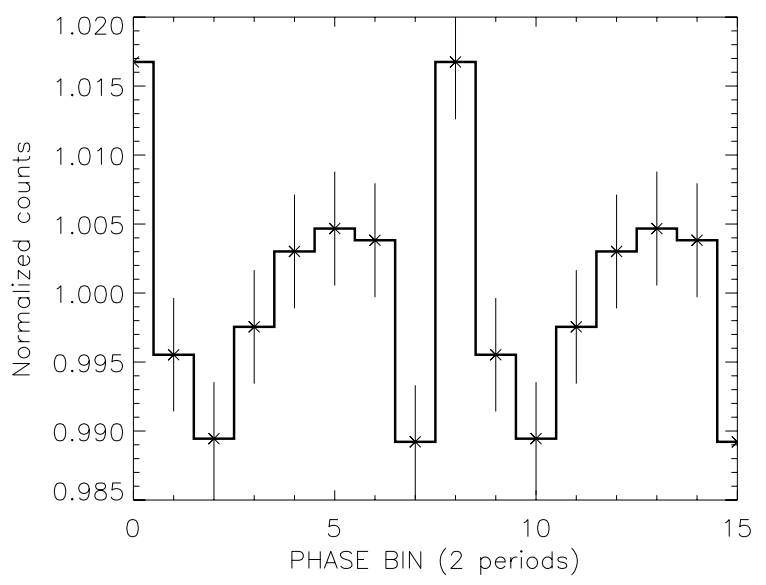

Fig. 4. LMC X-4 20-40 keV light curve folded at the best fit period of $13.497 \pm 0.005 \mathrm{~s}$.

band, the one with the highest signal-to-noise ratio. An additional selection criterion to improve our statistics was to choose only those pixels illuminated by the source for at least $75 \%$ of their surface. We applied an epoch-folding analysis to search in the range from $13.4 \mathrm{~s}$ to $13.6 \mathrm{~s}$, with 8 phase bins and 150 trial periods. This period range is much larger than the range of periods ever observed in this source (see Fig. 2 in Woo et al. 1996). We could not detect the pulsations in the 2003 data, which are affected by a higher background noise, having been taken at the beginning of the mission when the on board parameters of the instruments were not yet completely optimised. The search was more fruitful for the 2004 observation when pulsations were detected considering five time series together taken from orbit 152, starting at 53016.54105 MJD and ending at 53016.71887 MJD (total exposure $11.5 \mathrm{ks}$ ). Fitting the distribution of $\chi^{2}$ values versus trial period as described in Leahy (1987), we obtained a best-fit period of $13.497 \pm 0.005 \mathrm{~s}$. The folded light curve (Fig. 4) shows two peaks per phase, in contrast to the single broad peak measured between 15 and $37 \mathrm{keV}$ by Ginga (Woo et al. 1996) and with SAX between 23 and 30 keV (Naik \& Paul 2004). 


\section{Discussion}

\section{1. $L M C X-1$}

LMC X-1 is a well-known black hole candidate. Together with Cyg $\mathrm{X}-1$ and LMC X-3, it is the only persistent X-ray binary with an accurately determined mass function, strongly suggesting the presence of an accreting black hole (Hutchings et al. 1987). LMC X-1 spends most of the time in the socalled high/soft state, characterised by a spectrum modeled with a multi-temperature disk black body plus a power law component dominating above $10 \mathrm{keV}$ and a narrow emission line at $6.5 \mathrm{keV}$. The photon index of the power law component is usually around 3 (Nowak et al. 2001).

This is the first time that the high-energy flux of this source can be fully disentangled (see Fig. 1) from that of PSR B0540-69, which lies just 24.6' from it (see e.g. Haardt et al. 2001). We only detected LMC X-1 up to $\sim 80 \mathrm{keV}$ during the 2003 observation. The flux measured in 2003 (see Table 3) was one order of magnitude higher than the one measured by $S A X$ in the same energy range (Haardt et al. 2001) and a factor 5 larger than reported with RXTE (Nowak et al. 2001). This could be interpreted as transition of the source to its low/hard state. However, the photon index $(\sim 3)$ and the fact that the 2-10 keV flux, measured with the All Sky Monitor (ASM) on board RXTE (see Fig. 5), remained constant, indicate that the source was in high/soft state. Hence the 2003 observation represents the highest hard X-ray flux reported so far for the soft state of LMC X-1.

The non-detection of LMC X-1 in 2004 indicates that it became fainter by a factor $\sim 10$. The $3 \sigma$ upper limit on its flux in January 2004 is $2.6 \times 10^{-4} \mathrm{ph} \mathrm{cm}^{-2} \mathrm{~s}^{-1}$ in the $20-100 \mathrm{keV}$ band (assuming the same spectral shape as in the 2003 observation). This value is still compatible with the $S A X / \mathrm{PDS}$ measurement. The 2-10 keV ASM flux remained almost constant during both observations (see Fig. 5), indicating we are again measuring a soft state of the source. A similar behaviour, with large variations of the hard-X ray component independent of the soft component, has been observed in this source and in LMC X-3 using Ginga data (Ebisawa et al. 1989, 1993). This seems to indicate that there is a mechanism responsible for the variation of high-energy emission, which does not involve variations of the low-energy flux.

\section{2. $L M C X-3$}

LMC X-3 is another black hole candidate, similar in many respects to LMC X-1 (Nowak et al. 2001), with the main difference that it shows a higher degree of variability at soft X-rays. In fact it undergoes strong variations in luminosity associated with the cyclic transitions between its low/hard and its high/soft state. During the low/hard state, the flux dims at low energies, but grows at high energies, pivoting at $\sim 10 \mathrm{keV}$. The spectrum can then be represented by a single power law with photon index 1.7 (Boyd et al. 2000; Wilms et al. 2001).

LMC X-3 was not detected in our INTEGRAL observations. We can put a $3 \sigma$ upper limit on its flux $(20-100 \mathrm{keV})$ of $1.8 \times 10^{-4} \mathrm{ph} \mathrm{cm}^{-2} \mathrm{~s}^{-1}$ deduced from the first observation.

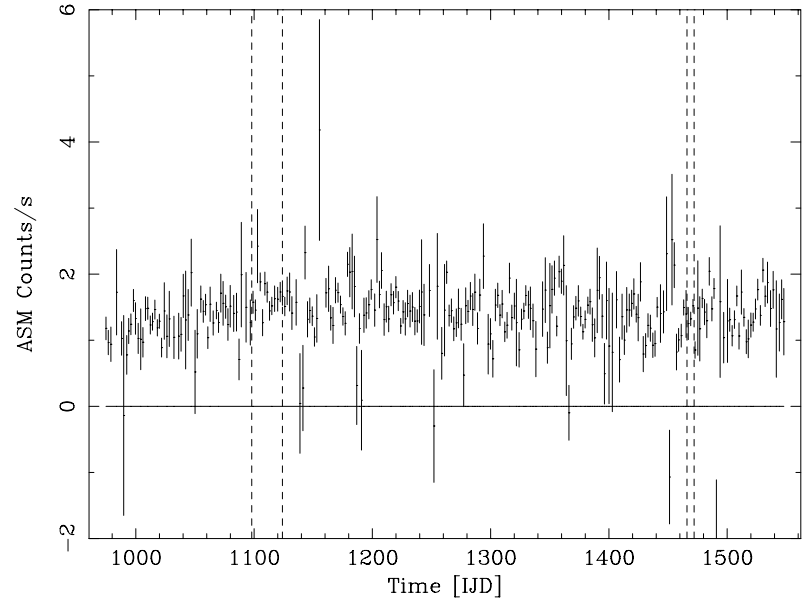

Fig. 5. ASM light curve (2 day-averages) of LMC $X-1$. The two INTEGRAL observations occurred in the periods delimited by vertical dashed lines.

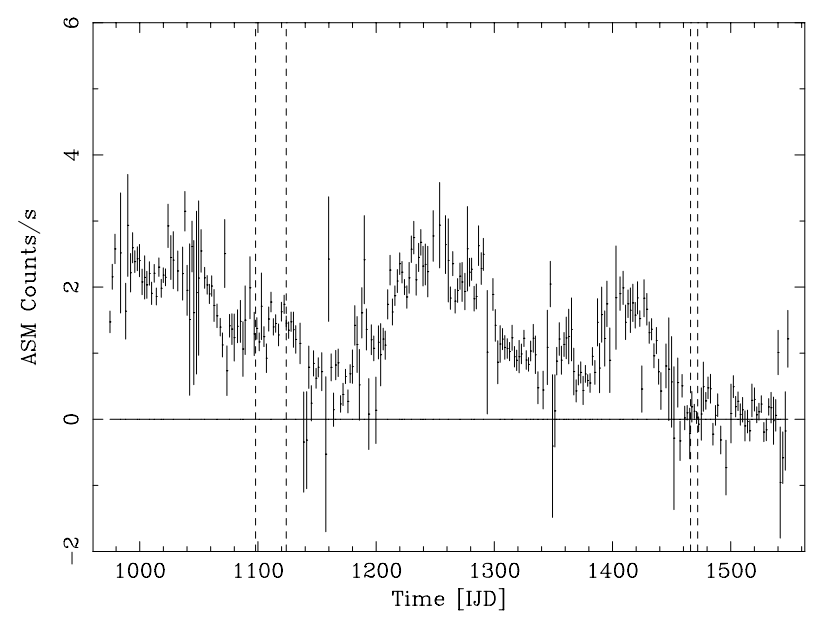

Fig. 6. ASM light curve (2 day-averages) of LMC X-3. The two INTEGRAL observations occurred in the periods delimited by vertical dashed lines.

Following Wilms et al. (2001), one can associate the minima of the ASM light curve with the hard states of the source. By comparing our data with the ASM (see Fig. 6), we can conclude that we did not observe the hard state of the source in 2003. In fact our first observation was close but did not exactly correspond to a minimum. Our non-detection can be explained by the source being in a soft state without a strong high energy component similar to what we observe during the second LMC X-1 observation and to what was reported for the March 1988 Ginga observation (Ebisawa et al. 1993).

The second INTEGRAL observation, on the other hand, corresponds to an extremely low X-ray state (no detection in the ASM light curve), close to quiescence, which is similar to the "off" states of transient black hole binaries. This picture is consistent with our upper limit $\left(\sim 3 \times 10^{-4} \mathrm{ph} \mathrm{cm}^{-2}\right)$ derived for the 2004 observation, which is lower than the extrapolation of the flux measured at lower energies with the PCA during low/hard states (Boyd et al. 2000). 


\section{3. $L M C X-4$}

LMC X-4 is a high-mass X-ray binary system composed of an O/B type star (Sanduleak \& Philip 1977), probably a mainsequence star (Woo et al. 1996), and a pulsating neutron star. It has an intense magnetic field (La Barbera et al. 2001), and it accretes material from the companion by stellar wind. As for Her $\mathrm{X}-1$, an accretion disk that is thicker in the outer regions and truncated at the Alfén radius by the magnetic pressure, is also present (Lang et al. 1981).

The disk emits thermal photons that contribute to the main soft (0.1-2 keV) component of the spectrum. Hard X-ray photons are instead produced by reprocessing in an external hot corona that surrounds the inner part of the disk: soft photons are, in general, Compton-inverse upscattered to higher energy. When the disk is edge-on, only the contribution due to the corona (Woo et al. 1996) is visible. This behaviour is confirmed by the absence of spectral variations between high and low state (see also Naik \& Paul 2003, 2004).

If the indication of resolved ingress duration derived from our data is confirmed, the corresponding size for the region emitting above $20 \mathrm{keV}$ is clearly not compatible with the size of the neutron star, confirming that the hard X-rays are produced in a hot scattering corona surrounding the accretion disk.

LMC X-4 is thought to be near the condition of spin equilibrium, which occurs when the spin period of the neutron star is close to the Keplerian period of the inner boundary of the accretion disk. This condition is reflected in a rather constant spin period evolution. In fact the latter has been studied for more than 20 years (see e.g. Woo et al. 1996; Levine et al. 2000; Naik \& Paul 2004) and has shown some variations but no particular spin-up or spin-down trend. Our measurement is still in the range of the reported periods for this source.

\subsection{PSR B0540-69}

One of the youngest rotation-powered pulsars, PSR B0540-69, was discovered in the X-rays by the Einstein Observatory (Seward et al. 1984). Its rotational characteristics $(P=50 \mathrm{~ms}$ and $\dot{P}=4.79 \times 10^{-13} \mathrm{~s} \mathrm{~s}^{-1}$ ) resemble those of the Crab pulsar and give a characteristic age of $\sim 1.6 \times 10^{3} \mathrm{yr}$. Like the $\mathrm{Crab}$, it is surrounded by a bright radio, optical, and X-ray synchrotron nebula.

The source was detected in our data up to $60 \mathrm{keV}$ with a marginal $(\sim 3 \sigma)$ detection in the $60-100 \mathrm{keV}$ band. This is the first time that a measure of the total flux from this pulsar and its nebula can be made at these energies. Up to now only the pulsed component could be measured with RXTE (de Plaa et al. 2003) up to $48 \mathrm{keV}$.

The flux we measured is about three times higher than the pulsed one measured by RXTE; see Fig. 7 . This means that the nebular and the unpulsed contributions at these energies would then be of the order of $50-75 \%$ of the total flux. This can be compared to what is reported in the same energy range for the Crab nebula, for which the nebula and unpulsed flux represent the $85 \%$ of the total flux (Kuiper et al. 2001).

The spectral index we derive is compatible, within the errors, with the one measured with $R X T E$. A softening at higher

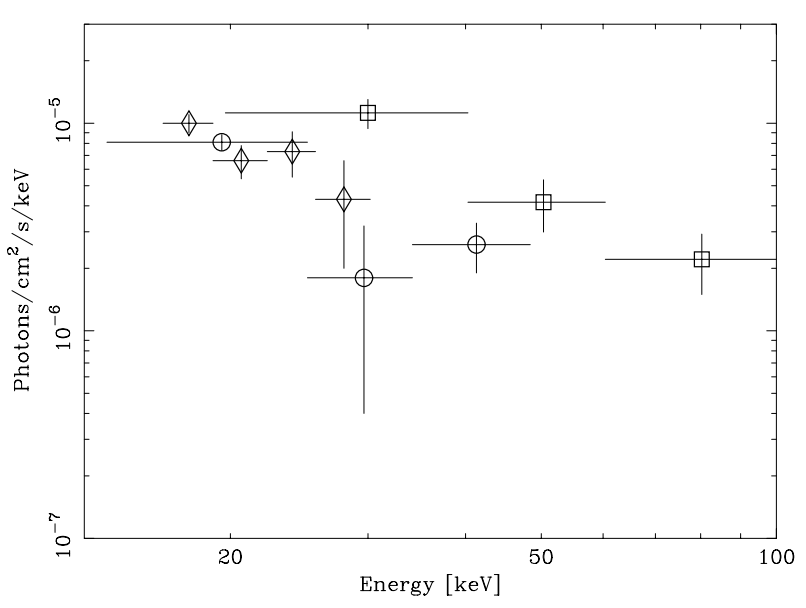

Fig. 7. PSR B0540-69 spectrum above $15 \mathrm{keV}$. The squares represent the total flux derived here with IBIS/ISGRI. The circles and the diamonds represent the pulsed emission measured with RXTE/HEXTE and RXTE/PCA, respectively (de Plaa et al. 2003).

energies has been reported for the HEXTE data on the pulsed emission: a spectral break in our data cannot be ruled out, due to the low statistics.

\subsection{EXO $0748-676$}

The low-mass X-ray binary (LMXB) EXO 0748-676, discovered with EXOSAT (Parmar et al. 1985), has been extensively studied in the soft X-ray band $(0.1-10 \mathrm{keV})$. It is a peculiar source, showing all types of temporal variability typical of the different kinds of LMXBs: it shows eclipses $\left(P_{\text {orb }}=3.82 \mathrm{~h}\right)$, dips, flares, and type I X-ray bursts indicating the presence of a neutron star in the system. The estimated distance of this source, lying outside the Galactic Plane at $b \simeq-20^{\circ}$, is $\sim 10 \mathrm{kpc}$, and the mass of the companion is estimated as $\sim 0.5 M_{\odot}$ (Gottwald et al. 1986; Parmar et al. 1986). A physical model for EXO 0748-676 was proposed by Church et al. (1998): the blackbody emission seen at low energies originates from the neutron star and an extended Comptonised emission from the Accretion Disc Corona (ADC) causes the cutoff power-law component measured in the hard X-ray band. Recent XMM-Newton results based on the absence (Bonnet-Bibaud et al. 2001) or presence (Homan et al. $2003)$ of eclipses in the soft energy band $(E<2 \mathrm{keV})$ seem to indicate that the dimensions of the Comptonised region vary and, in general, that the source has different states reflecting different geometries of the system.

The only hard X-ray observation of this source was performed by BeppoSAX in October 1998. The source was detected in the PDS up to $\sim 100 \mathrm{keV}$ (Sidoli et al. 2005). A broad-band $(0.1 \mathrm{keV}-100 \mathrm{keV})$ persistent (non-dip, nonburst) spectrum was obtained. They derived a $20-100 \mathrm{keV}$ flux of $3.68 \times 10^{-10} \mathrm{erg} \mathrm{cm}^{-2} \mathrm{~s}^{-1}$ which is fully compatible with the flux derived here. Also the cutoff energy of $48 \mathrm{keV}$ found in the SAX observation agrees with our data. This indicates that, despite the large flux variations found in the soft X-rays, the hard component seems to be more stable. 


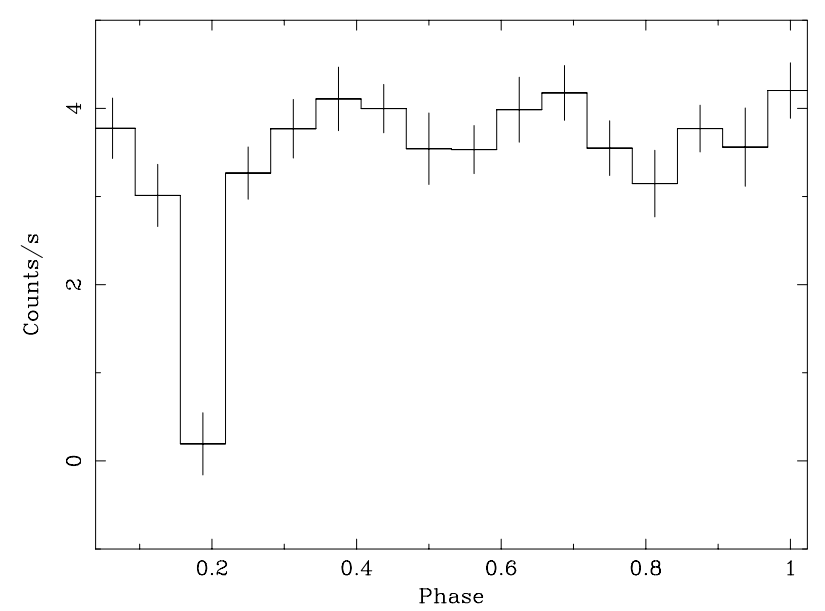

Fig. 8. IBIS/ISGRI light curve of EXO 0748-676 (20-100 keV) folded at the orbital period. The count rate is corrected for vignetting.

We extracted the light curve (20-100 keV) of EXO 0748-676 with $600 \mathrm{~s}$ time bins (corresponding roughly to the eclipse duration at lower energies) and folded it at the orbital period value. The resulting light curve, Fig. 8, clearly indicates the eclipse. However, the low statistics due to the fact that EXO 0748-676 was detected at a very large off-axis angle do not allow us to perform more detailed analysis. The eclipse above $20 \mathrm{keV}$ is consistent with being total, indicating that the angular size of the companion star is greater than that of the hard X-ray emission region.

\subsection{IRAS $04575-7537$}

The AGN IRAS 04575-7537 is associated with a rather bright barred spiral Galaxy at redshift $z=0.0184$ and it shows strong infrared emission (Hewitt \& Burbidge 1991). It was first detected in the X-ray range with HEAO-1, and later observed with ROSAT, Ginga, and ASCA (Vignali et al. 1998). It is classified as a Seyfert 2 galaxy.

In our observations it was quite faint $(\sim 3 \mathrm{mCrab})$ and detectable up to $\sim 80 \mathrm{keV}$ in the IBIS/ISGRI mosaic. We derived two spectral points and an upper limit, which represents the first measurement of this AGN at these energies. The flux is about $1.5 \times 10^{-11} \mathrm{erg} \mathrm{cm}^{-2} \mathrm{~s}^{-1}(20-100 \mathrm{keV})$, which implies a $20-100 \mathrm{keV}$ luminosity of $1.2 \times 10^{43} \mathrm{erg} \mathrm{s}^{-1}\left(H_{0}=70\right.$, $\left.\lambda_{0}=0.7\right)$.

\subsection{New sources}

The source IGR J05319-6601 is located at a position consistent with the LMC. It is at an angular distance of $21^{\prime}$ from LMC X-4, and their point-spread functions partially overlap; therefore, its significance and flux are likely to be influenced by the brighter source. Within its error box $\left(\sim 3.5^{\prime}\right)$ there are at least 7 faint unidentified soft X-ray sources, discovered during different X-ray surveys of the LMC. Hence we cannot exclude that we are measuring the blended emission from different unresolved sources. The $20-40 \mathrm{keV}$ luminosity we derive for this source, supposing it is located in the LMC, is of the order of

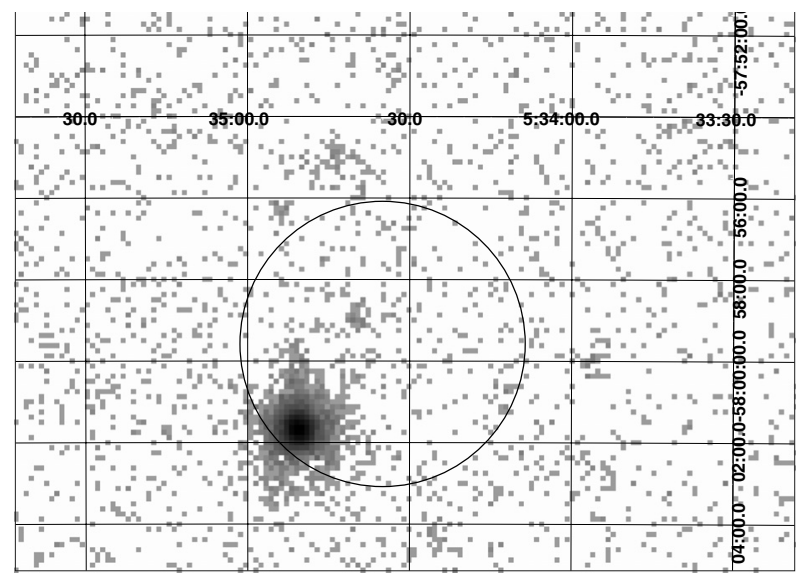

Fig. 9. ROSAT/PSPC 0.2-2 keV image of TW Pic. The circle represents the error box associated with IGR J05346-5759.

$10^{36} \mathrm{erg} \mathrm{s}^{-1}$. This value and the fact that IGR J05319-6601 is close to the centre of the galaxy suggest that we are dealing with a binary system.

IGR J05009-7044 is possibly the same source recently reported as IGR J05007-7047 for which a Chandra X-ray counterpart has been found (Halpern 2005). The Chandra source is optically identified with the bright blue star USNO-B1.00192-00057570 (Monet et al. 2003). This suggests that IGR J05009-7044 could be a high mass X-ray binary (HMXB) in the LMC. In this case it would have a $20-40 \mathrm{keV}$ luminosity of $\sim 7 \times 10^{35} \mathrm{erg} \mathrm{s}^{-1}$. However, inside the error box of IGR J05009-7044 there is also a radio emitting galaxy (SUMSS J050059-704225, 10 mJy at $843 \mathrm{Mhz}$, Mauch et al. 2003), so we cannot exclude the possibility that the INTEGRAL source is instead an AGN.

All the other new sources we discovered are at positions that are not consistent with the LMC. IGR J05346-5759 is spatially coincident with TW Pic (see Fig. 9), a cataclysmic variable of the DQ Her type (Norton et al. 2000). We analysed an archival ROSAT/PSPC observation of this source. The observation is $\sim 10 \mathrm{ks}$ long and was performed on April 10, 1992. The source spectrum is well-fitted with an absorbed power law model. We derive a photon index $\Gamma=1.3 \pm 0.2$ and a hydrogen column density $N_{\mathrm{H}}=(1.2 \pm 0.5) \times 10^{20} \mathrm{~cm}^{-2}$, with an unabsorbed $0.5-2 \mathrm{keV}$ flux of $3 \times 10^{-12} \mathrm{erg} \mathrm{cm}^{-2} \mathrm{~s}^{-1}$. The extrapolation of this spectrum to the $20-40 \mathrm{keV}$ energy band yields a flux of $1.3 \times 10^{-11} \mathrm{erg} \mathrm{cm}^{-2} \mathrm{~s}^{-1}$, which is fully compatible with the $\sim 1 \times 10^{-11} \mathrm{erg} \mathrm{cm}^{-2} \mathrm{~s}^{-1}$ value derived from the IBIS data.

IGR J03532-6829 can be associated with 1RXS J035257.7-683120, which has been discovered in the X-rays by the Einstein satellite (Elvis et al. 1992). It has been detected also with RXTE in the $8-20 \mathrm{keV}$ band (Revnivtsev et al. 2004) and is very likely associated with the AGN PKS 0352-686. IGR J06239-6052 is coincident with a radio galaxy (SUMSS J062346-605222, Mauch et al. 2003), making it an AGN candidate. 


\section{Conclusions}

The INTEGRAL survey of the LMC allowed us to study the hard X-ray emission of some of the sources of this galaxy for the first time. Among the sources of the LMC we detected and studied LMC X-1, LMC X-4, and PSR B0540-69. We could not detect LMC X-3 during our observations, being in its soft state during the first one and close to quiescence in the second one. Our data on the eclipses of the accreting X-ray pulsar LMC $X-4$ suggest that the size of the high-energy emission region is $\sim 3 \times 10^{10} \mathrm{~cm}$.

Also for the first time we were able to disentangle the highenergy emission of PSR B0540-69, for which we measured the total (pulsar+nebula) spectrum, from the one of LMC X-1, for which we measured the flux and long-term variability.

Several serendipitous sources were detected during our survey: the brightest ones are EXO 0748-676, IRAS 04575-7537, and SMC X-1. We derived their fluxes and spectral parameters, where possible. The eclipses of EXO 0748-676 were reported for the first time at energies above $\sim 10 \mathrm{keV}$.

In addition we reported the discovery of 5 new faint highenergy sources. Two of them are most likely associated with AGNs. One represents the first high-energy detection of the cataclysmic variable TW Pic, and the last two are potentially located in the LMC. One (IGR J05319-6601) has no clear association and could be due to the blended emission of several sources, while the other (IGR J05009-7044) may be an HMXB.

Acknowledgements. We acknowledge the Italian Space Agency's financial and programme's support via contract I/R/046/04.

\section{References}

Arnaud, K. A. 1996, Astronomical Data Analysis Software and Systems V, ASP Conf. Ser., 101, 17

Bonnet-Bibaud, J. M., Haberl, F., Ferrando, P., Bennie, P. J., \& Kendziorra, E. 2001, A\&A, 365, L282

Boyd, P. T., Smale, A. P., \& Homan, J. 2000, ApJ, 542, L127

Church, M. J., Balucinska-Church, M., Dotani, T., \& Asai, K. 1998, ApJ, 504, 516

Courvoisier, T. J.-L., Walter, R., Beckmann, V., et al. 2003, A\&A, 411 L53

Ebisawa, K., Mitsuda, K., \& Inoue, H. 1989, PASJ, 41, 519

Ebisawa, K., Makino, F., Mitsuda, K., et al. 1993, ApJ, 403, 684

Elvis, M., et al. 1992, ApJS, 80, 527

Finoguenov, A., Gilfanov, M., Grebenev, S., et al. 1993, AstL, 19, 69

Gottwald, M., Haberl, F., Parmar, A. N., \& White, N. E. 1986, ApJ, 308, 213

Grebenev, S., Sunyaev, R., Pavlinsky, M., et al. 1991, AstL, 17, 310
Haardt, F., Galli, M. R., Treves, A., et al. 2001, ApJS, 133, 187

Haberl, F., \& Pietsch, W. 1999, A\&AS, 139, 277

Haberl, F., Dennerl, K., \& Pietsch, W. 2003, A\&A, 406, 471

Halpern, J. P. 2005, ATel, 572

Hewitt, A., \& Burbidge, G. 1991, ApJSS, 75, 297

Homan, J., Wijnands, R., \& van den Berg, M. 2003, A\&A, 412, 799

Hutchings, J. B., Crampton, D., Cowley, A. P., Bianchi, L., \& Thompson, I. B. 1987, AJ, 94, 340

Kuiper, L., Hermsen, W., Cusumano, G., et al. 2001, A\&A, 2001, 378, 918

La Barbera, A., Burderi, L., Di Salvo, T., Iaria, R., \& Robba, N. R. 2001, ApJ, 553, 375

Lang, F. L., Levine, A. M., Bautz, M., et al. 1981, ApJ, 246, L21

Leahy, D. A. 1987, A\&A, 180, 275

Lebrun, F., Leray, J. P., Lavocat, P., et al. 2003, A\&A, 411, L141

Levine, A., Rappaport, S., Putney, A., Corbet, R., \& Nagase, F. 1991, ApJ, 381, 101

Levine, A. M., Rappaport, S. A., \& Zojcheski, G. 2000, ApJ, 541, 194

Long, K. S., Helfand, D. J., \& Grabelsky, D. A. 1981, ApJ, 248, 925

Markert, T. H., \& Clark, G. W. 1975, ApJ, 196, L55

Mauch, T., Murphy, T., Buttery, H. J., et al. 2003, MNRAS, 342, 1117

Mereghetti, S., Götz, D., Mirabel, I. F., \& Hurley, K. 2005, A\&A, 433, L9

Monet, D. G., Levine, S. E., Canzian, B., et al. 2003, AJ, 125, 984

Naik, S., \& Paul, B. 2003, A\&A, 401, 265

Naik, S., \& Paul, B. 2004, ApJ, 600, 351

Norton, A. J., Beardmore, A. P., Retter, A., \& Buckley, D. A. H. 2000, MNRAS, 312, 362

Nowak, M. A., Wilms, J., Heindl, W. A., et al. 2001, MNRAS, 320, 316

Parmar, A. N., White, N. E., Giommi, P., et al. 1985, IAU Circ., 4039

Parmar, A. N., White, N. E., Giommi, P., \& Gottwald, M. 1986, ApJ, 308, 199

Paul, B., \& Kitamoto, S. 2002, JApA, 23, 33

de Plaa, J., Kuiper, L., \& Hermsen, W. 2003, A\&A, 400, 1013

Revnivtsev, M., Sazonov, S., Jahoda, K., \& Gilfanov, M. 2004, A\&A, 418, 927

Seward, F. D., Harnden, F. R., \& Helfand, D. J. 1984, 287, L19

Sidoli, L., Parmar, A. N., \& Oosterbroek, T. 2005, A\&A, 429, 291

Sanduleak, N., \& Philip, A. G. D. 1977, IAUC Circ., No 3023

Sasaki, M., Haberl, F., Pietsch, W., et al. 2000, A\&AS, 143, 391

Sunyaev, R., Kaniovsky, A., Efremov, V., et al. 1987, Nature, 330, 227

Sunyaev, R., Gilfanov, M., Churazov, E., et al. 1990, AstL, 16, 124

Treves, A., Galli, M., Haardt, F., et al. 2000, AdSpR, 25, 437

Ubertini, P., Lebrun, F., Di Cocco, G., et al. 2003, A\&A, 411, L131

Vignali, C., Comastri, A., Stirpe, G. M., et al. 1998, A\&A, 333, 411

White, N. E. 1978, Nature, 271, 38

Wilms, J., Nowak, M. A., Pottschmidt, K., et al. 2001, MNRAS, 320, 327

Woo, J. W., Clark, G. W., Levine, A. M., et al. 1996, ApJ, 467, 811

Winkler, C., Courvoisier, T. J.-L., Di Cocco, G., et al. 2003, A\&A, 411, L1 